\title{
The risk factors and outcomes of preoperative hepatic dysfunction in patients who receive surgical repair for acute type $A$ aortic dissection
}

\author{
Zhigang Wang ${ }^{1}$, Tao Chen ${ }^{1}$, Min Ge ${ }^{1}$, Cheng Chen ${ }^{1}$, Lichong Lu ${ }^{1}$, Lifang Zhang ${ }^{2}$, Dongjin Wang ${ }^{1}$ \\ ${ }^{1}$ Department of Cardio-thoracic Surgery, Affiliated Drum Tower Hospital, Medical School of Nanjing University, Nanjing, China; ${ }^{2}$ Department of \\ Psychiatry, The First Affiliated Hospital, Zhengzhou University, Zhengzhou, China \\ Contributions: (I) Conception and design: D Wang; (II) Administrative support: D Wang, M Ge; (III) Provision of study materials or patients: Z \\ Wang, T Chen, M Ge, C Chen; (IV) Collection and assembly of data: Z Wang, T Chen, L Lu; (V) Data analysis and interpretation: Z Wang, L \\ Zhang; (VI) Manuscript writing: All authors; (VII) Final approval of manuscript: All authors. \\ Correspondence to: Dongjin Wang. Department of Cardio-thoracic Surgery, Affiliated Drum Tower Hospital, Medical School of Nanjing University, \\ Zhongshan Road 321, Nanjing 210008, China. Email: glyywdj@163.com.
}

\begin{abstract}
Background: Hepatic dysfunction (HD) is a common complication that can occur after surgical repair of acute type A aortic dissection (ATAAD) and is associated with poor prognosis. However, the incidence of early preoperative HD and the associated risk factors in patients with ATAAD have not been fully elucidated.

Methods: A total of 984 ATAAD patients who received surgical repair within 48 hours of symptom onset at our department from January 2014 to December 2019 were retrospectively analyzed. Patients were divided into the non-HD group and the HD groups according to the Model of End-Stage Liver Disease (MELD) score before surgery. The clinical parameters and clinical outcomes of the 2 groups were collected and compared.

Results: Preoperative HD was detected in 268 patients $(27.2 \%)$. The incidence of in-hospital complications, including the need for dialysis $(34.0 \%$ vs. $9.2 \% ; \mathrm{P}<0.001)$, was significantly higher in patients with HD compared to patients without HD (69.8\% vs. $51.0 \%$; $<<0.001)$. Patients with HD had a higher 30-day mortality rate compared to patients without HD (20.1\% vs. 8.4\%; $\mathrm{P}<0.001)$. Multivariate analysis demonstrated that preoperative cardiac tamponade, preoperative serum creatinine levels, and serum troponin T levels upon admission were independent predictors for preoperative HD in patients with ATAAD. Interestingly, even though preoperative HD was associated with an increased 30-day mortality rate, it did not significantly affect the long-term mortality rate (log-rank $\mathrm{P}=0.259$ ).

Conclusions: Early HD before surgery was commonly observed in patients with ATAAD and was associated with increased in-hospital complications after surgery, but did not significantly affect long-term survival.
\end{abstract}

Keywords: Hepatic dysfunction (HD); risk factors; type A aortic dissection; outcomes

Submitted Jun 24, 2021. Accepted for publication Sep 17, 2021.

doi: $10.21037 /$ jtd-21-1051

View this article at: https://dx.doi.org/10.21037/jtd-21-1051

\section{Introduction}

Acute type A aortic dissection (ATAAD) is a rare but often catastrophic disease with an incidence of 3.5 to 6 per 100,000 (1). It often progresses rapidly and is accompanied by life-threatening complications such as aortic rupture, cardiac tamponade, coronary obstruction, and visceral ischemia that require emergent surgical management. Delay with ATAAD repair increases the risk of mortality by $1 \%$ per hour (2). Despite recent advances in medical management and surgical techniques, ATAAD is still associated with 
significant morbidity and mortality (10-20\%) $(3,4)$.

For the past 30 years, the Child-Turcotte-Pugh (CTP) classification has been applied clinically to evaluate the severity of hepatic dysfunction (HD) (5). However, recent studies have suggested that the Model of End-Stage Liver Disease (MELD) is a more robust score and a better index compared to the CTP classification (6). Irrespective of the etiology of preoperative liver disease, patients with HD who require surgery are generally at greater risk of perioperative complications than those with normal liver function (7). Notably, cardiac surgery in patients with HD has been associated with an even higher mortality rate compared to other surgical procedures (8). HD is commonly observed after aortic surgery for ATAAD and is often associated with increased in-hospital mortality (9). A recent study showed that preoperative hepatic function could effectively predict postoperative mortality and morbidity after cardiac surgery (10). Therefore, careful evaluation of the severity of preoperative $\mathrm{HD}$ is important to identify patients with increased risks. However, there is a paucity of data regarding early preoperative HD and its clinical and prognostic relevance in patients with ATAAD. We hypothesized that preoperative HD is commonly observed and associated with prognosis after ATAAD repair. This study examined the incidence and predictors of early preoperative HD using MELD scores. Furthermore, the effects of preoperative HD on in-hospital and long-term prognosis in patients receiving ATAAD surgery were evaluated.

We present the following article in accordance with the STROBE reporting checklist (available at https://dx.doi. org/10.21037/jtd-21-1051).

\section{Methods}

\section{Study population}

ATAAD patients who were diagnosed by computed tomography angiography (CTA) and who received emergency surgery in our hospital were retrospectively enrolled in this study. Patients with dialysis dependent endstage renal failure, recurrent aortic dissection, incomplete data (lack of pre- and postoperative CTA test), and recent warfarin treatment were excluded from the study. Clinical variables including patient demographics, medical histories, and clinical outcomes, including both in-hospital complications and mortality, were collated. This study was approved by the Ethics Committee of the Nanjing Drum Tower Hospital (no. BL2014004). Individual patient consent was waived due to the retrospective nature of this study. The study was conducted in accordance with the Declaration of Helsinki (as revised in 2013).

\section{Definitions}

The MELD score was calculated for each patient using the following standard formula published by the Mayo Clinic College of Medicine: MELD $=11.2 \times \ln$ (international normalized ratio $[\mathrm{INR}])+3.78 \times \ln ($ serum total bilirubin $[\mathrm{mg} / \mathrm{dL}])+9.57 \times \ln ($ serum creatinine $[\mathrm{mg} / \mathrm{dL}])+6.43(11)$. Any variable with a value less than 1 was assigned a value of 1 to avoid negative scores, and values exceeding 4 for creatinine were all replaced by 4 . The minimum possible MELD score was 6.43 .

A MELD cutoff point of 13 has been used in several previous studies investigating mortality in patients with cirrhosis undergoing cardiac surgery $(12,13)$. However, another study investigating the impact of MELD scores on prognosis of heart failure used a cutoff point of 12 based on over 300 patients undergoing heart transplant evaluation (14). This was similar to another recent research that predicted postoperative mortality and morbidity after cardiac surgery which also applied a cutoff value of 12 (10). Therefore, in this current study, a MELD score of 12 was used as a cutoff value to identify patients with HD. All patients were separated based on their preoperative score and assigned into the non-HD group (MELD < 12) and the HD group (MELD $\geq 12$ ).

Coronary malperfusion was defined by electrocardiograph ST changes or determined by the surgeons according to the patients' coronary artery status during the operation. Hypotensive shock was defined as systolic blood pressure less than $90 \mathrm{mmHg}$, regardless of the etiology. The 30-day mortality was defined as death within 30 days after ATAAD repair.

\section{Total arch replacement (TAR) surgery}

TAR surgery plus frozen elephant trunk (FET) has been widely used in China, especially for patients with tears in the aortic arch, Marfan syndrome, other connective tissue disorders, and aneurysm dilation of the arch or supraarch vessels. During the TAR procedure, after FET was inserted into the true lumen of the descending thoracic aorta, the remaining aortic arch and descending aorta were anastomosed with an artificial vessel. Aortic arch operations were performed under deep hypothermic circulatory 


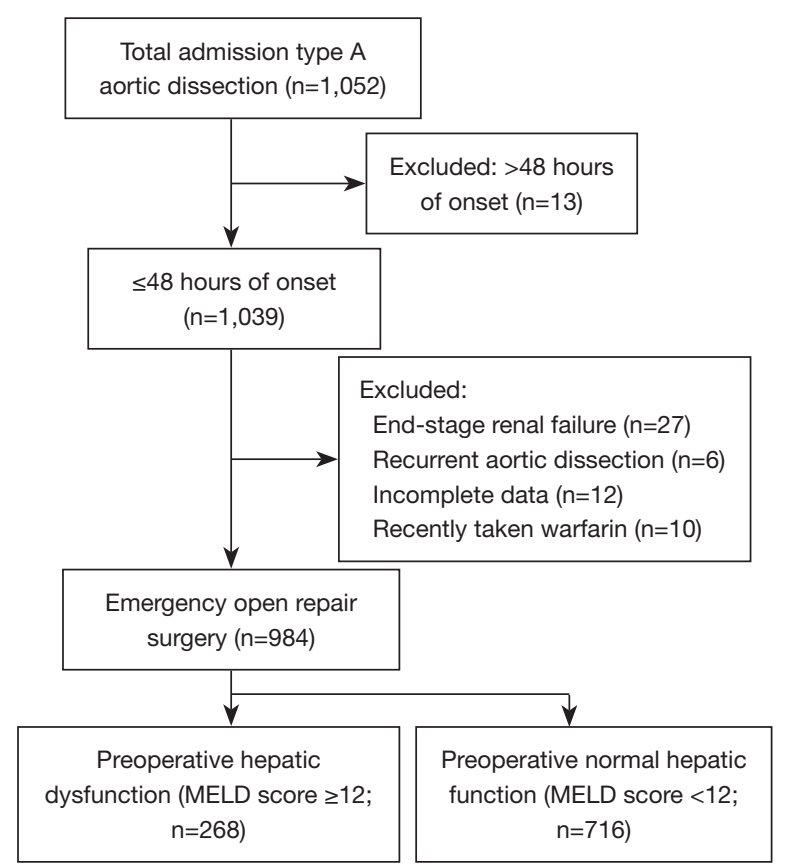

Figure 1 The patient selection process. MELD, Model of EndStage Liver Disease.

arrest with selective cerebral perfusion and open distal anastomosis.

\section{Statistical analysis}

Categorical variables are presented as frequencies with percentages. Continuous variables are presented as mean \pm standard deviation or median (interquartile range). Chisquare or Fisher's test was used to compare categorical variables, and the $t$-test or Mann-Whitney $U$ test was used for continuous variables as appropriate. To reduce selection bias, one-to-one pair matching was performed using nearest neighbor matching without replacement within 0.2 standard deviations of the logit of the propensity score as a caliper width.

To determine independent predictors of preoperative $\mathrm{HD}$, stepwise multivariate logistic regression analyses were performed using variables with $\mathrm{P}$ value $<0.3$ identified in the univariate analyses. To detect risk factors for mortality, multivariate logistic regression models were used. Receiver operating characteristic (ROC) curves were designed to identify cutoff values of risk factors for preoperative HD. The prognostic value of the MELD scores was determined by calculating the area under the curve (AUC) from the ROC curve analysis for postoperative dialysis and the 30-day morbidity. The best possible cutoff point was defined as the highest Youden Index [(specificity + sensitivity) - 1]. Crude survival excluding in-hospital deaths was estimated with the Kaplan-Meier method. Differences in survival rate between groups were analyzed with log-rank tests. A P value less than 0.05 was considered statistically significant. All analyses were conducted using SPSS software (version 25.0 IBM Corp, Armonk, NY, USA).

\section{Results}

The selection process and number of selected patients are shown in Figure 1. A total of 1,052 consecutive patients who received open surgery for ATAAD at Nanjing Drum Tower Hospital from January 2014 to December 2019 were screened for this study. As this study focused on early preoperative HD, patients admitted to our hospital more than 48 hours after the onset of symptoms were excluded $(n=13)$. In addition, patients with end-stage renal failure maintained on chronic dialysis $(n=27)$, patients with recurrent aortic dissection $(n=6)$, or patients with incomplete data $(\mathrm{n}=12)$ were excluded from the study. Patients who were administered warfarin before surgery $(n=10)$ were also excluded from the investigation. Finally, a total of 984 patients with a median age of 53 years (range, 19 to 87 years) were included in this analysis.

In this study cohort, preoperative HD was a common condition in ATAAD patients. As shown in Table 1, among the 984 enrolled patients, 268 (27.2\%) presented with HD. There were no significant differences in terms of medical history characteristics between patients with HD and patients without HD. The time from onset of chest pain to hospital admission was comparable between the HD group and the non-HD group, as was the liver blood supply condition between the 2 groups. Among the 810 patients (82.3\%) characterized with DeBakey type I aortic dissection, 215 patients developed preoperative HD. No differences were found between type I and type II aortic dissections. Univariate analysis identified significant differences in the prevalence of preoperative coronary ischemia, hypotension, and pericardial tamponade between the HD and nonHD groups. There were significant differences in certain serum parameters between the 2 groups, including the levels of white blood cell, hemoglobin, platelet, fibrinogen, C-reactive protein, D-dimer, albumin, troponin $\mathrm{T}(\mathrm{Tn} \mathrm{T})$, alanine transaminase, aspartate transaminase, blood urea nitrogen, creatinine, bilirubin, and INR.

Patients in the HD group had a significantly higher 
Table 1 Comparison of preoperative variables

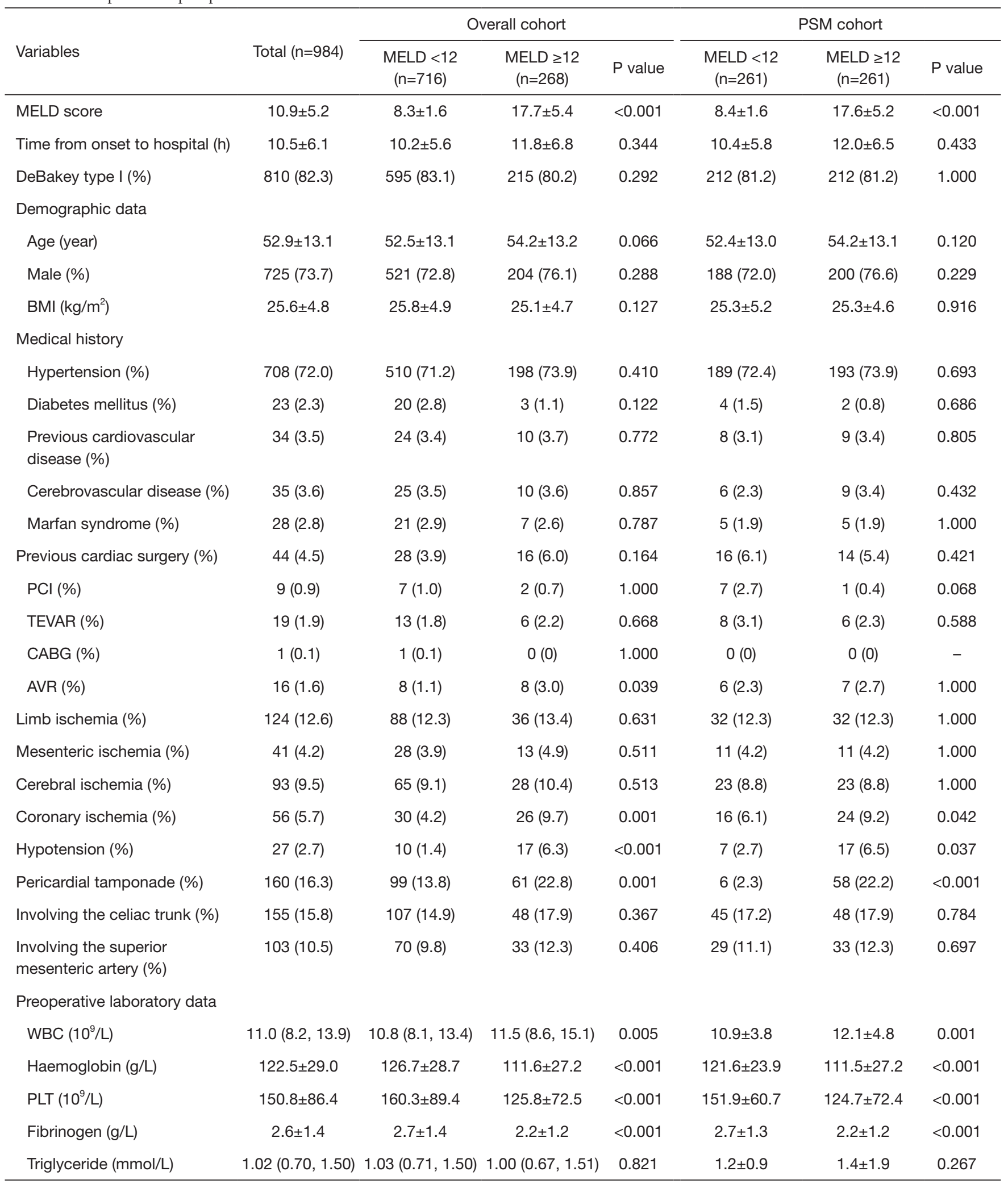

Table 1 (continued) 
Table 1 (continued)

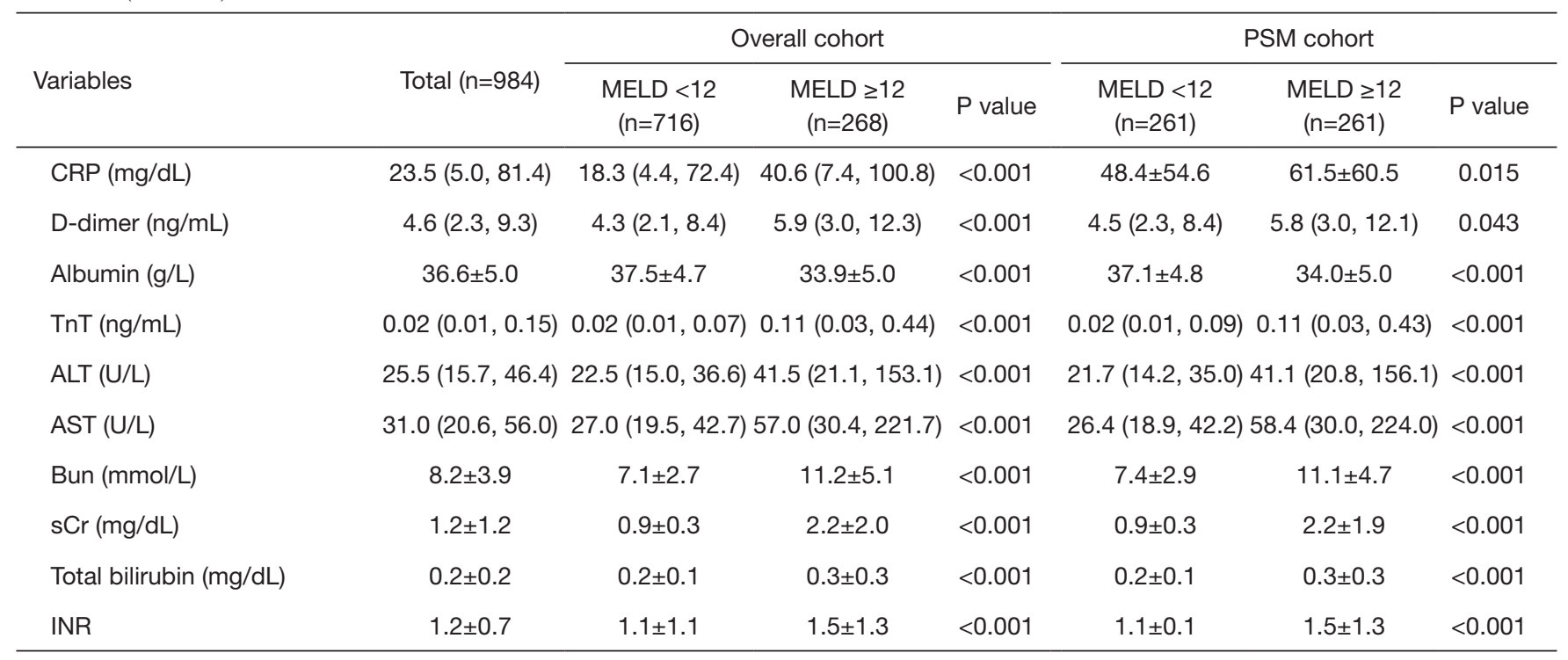

$\mathrm{P}$ values indicate differences between non-HD patients and HD patients. $\mathrm{P}<0.05$ was considered statistically significant. Data presented as $\mathrm{n}(\%)$, median (IQR), or mean \pm standard deviation. BMI, body mass index; WBC, white blood cell; SCr, serum creatinine; PLT, platelet; ALB, albumin; CRP, c-reactive protein; INR, international normalized ratio; PSM, propensity score matching.

incidence of in-hospital complications compared to those without HD (69.8\% vs. $51.0 \%, \mathrm{P}<0.001$; Table 2). In particular, drainage volume 24 hours after surgery, mechanical ventilation duration, incidence of tracheostomy, and dialysis, were all significantly increased in the HD group compared to the non-HD group. The incidence of other complications such as stroke, paraplegia, and total hospital stay time showed no significant difference between the 2 groups. The 30 -day mortality rate was $20.1 \%$ and $8.4 \%$ in patients with $\mathrm{HD}$ and without HD, respectively, and the difference was significant $(\mathrm{P}<0.001$; Table 2$)$. The duration of intensive care unit stay was also significantly prolonged in patients with HD compared to patients without $\mathrm{HD}(\mathrm{P}<0.001$; Table 2).

After propensity score matching, a total of 522 patients (261 pairs) were further analyzed. There were significant differences between the HD and the non-HD patients regarding the 30 -day mortality rate $(18.1 \%$ vs. $11.5 \%$; $\mathrm{P}=0.006$ ), as well as other variables such as preoperative coronary ischemia, hypotension upon admission, and ICU stay time (Table 1 and Table 2).

After adjusting for baseline variables, the data indicated that pericardial tamponade $[$ odds ratio $(\mathrm{OR})=2.000$; 95\% confidence interval (CI), 1.299-3.018; $\mathrm{P}=0.002$ ], preoperative serum creatinine (sCr) levels $(\mathrm{OR}=1.045 ; 95 \%$
CI, 1.037-1.054; $\mathrm{P}<0.001$ ), and TnT levels at admission (OR $=1.884 ; 95 \%$ CI, 1.395-2.544; $\mathrm{P}<0.001)$ were independent predictors for preoperative HD (Table 3). Furthermore, coronary ischemia was a significant risk factor for operative mortality $(\mathrm{OR}=32.630$; 95\% CI, 6.283-169.450; $\mathrm{P}<0.001$; Table 4). However, the MELD score was not a significant risk factor for operative mortality (Table 4). After the MELD score was included in the logistic regression model as an independent variable, elevated MELD score (OR $=1.128 ; 95 \% \mathrm{CI}, 1.029-1.236 ; \mathrm{P}=0.010)$ was identified as a risk factor for postoperative 30-day mortality (Table 5).

ROC curves were applied to evaluate the cutoff values of risk factors for preoperative HD and the cutoff values of the MELD score as predictive factors for dialysis and 30-day mortality. The cutoff value of TnT upon admission for preoperative HD was determined to be $0.051 \mathrm{ng} / \mathrm{mL}$, with $65.2 \%$ sensitivity and $72.2 \%$ specificity. The AUC for TnT was 0.724 (95\% CI, 0.688-0.760; $\mathrm{P}<0.001$; Figure 2). A MELD score of 11.0 yielded an AUC value of 0.743 (95\% CI, 0.697-0.788; $\mathrm{P}<0.001)$ with a $68.2 \%$ sensitivity and $71.5 \%$ specificity for predicting postoperative dialysis (Figure 3). In addition, a MELD score of 11.7 had a sensitivity of $67.5 \%$ and a specificity of $62.0 \%$ for predicting hospital mortality and the AUC for the MELD score was 0.682 (95\% CI, 0.630-0.735; $\mathrm{P}<0.001$ ). 
Table 2 Comparison of operative and postoperative variables

\begin{tabular}{|c|c|c|c|c|c|c|c|}
\hline Variables & Total $(n=984)$ & \multicolumn{3}{|c|}{ Overall cohort } & \multicolumn{3}{|c|}{ PSM cohort } \\
\hline CABG (\%) & $57(5.8)$ & $44(6.1)$ & $13(4.9)$ & 0.439 & $20(7.7)$ & $13(5.0)$ & 0.208 \\
\hline DHCA time (min) & $29.3 \pm 12.8$ & $29.7 \pm 13.0$ & $28.4 \pm 12.4$ & 0.177 & $27.7 \pm 12.7$ & $28.6 \pm 12.3$ & 0.424 \\
\hline Lowest temperature $\left({ }^{\circ} \mathrm{C}\right)$ & $20.6 \pm 3.1$ & $20.3 \pm 4.2$ & $20.8 \pm 2.9$ & 0.364 & $20.4 \pm 3.9$ & $20.9 \pm 3.6$ & 0.388 \\
\hline \multicolumn{8}{|l|}{ Root procedure } \\
\hline \multicolumn{8}{|l|}{ Distal surgical technique } \\
\hline Hemi-arch replacement (\%) & $210(21.3)$ & $151(21.1)$ & $59(22.0)$ & 0.752 & $116(44.4)$ & $56(21.5)$ & $<0.001$ \\
\hline $\begin{array}{l}\text { Total arch + frozen elephant trunk } \\
(\%)\end{array}$ & $467(47.5)$ & $336(46.9)$ & $131(48.9)$ & 0.585 & $137(52.5)$ & $129(49.4)$ & 0.484 \\
\hline Arch fenestrated stent graft (\%) & $308(31.3)$ & $223(31.3)$ & $85(31.7)$ & 0.863 & $127(48.7)$ & $80(30.7)$ & $<0.001$ \\
\hline Postoperative complications (\%) & $552(56.1)$ & $365(51.0)$ & $187(69.8)$ & $<0.001$ & $150(57.5)$ & $184(70.5)$ & $<0.001$ \\
\hline Re-exploration for bleeding (\%) & $39(4.0)$ & $17(2.4)$ & $22(8.2)$ & $<0.001$ & $6(2.3)$ & $22(8.4)$ & 0.002 \\
\hline Deep sternal wound infection (\%) & $13(1.3)$ & $8(1.1)$ & $5(1.9)$ & 0.532 & $3(1.1)$ & $5(1.9)$ & 0.724 \\
\hline $\begin{array}{l}\text { Drainage volume } 24 \mathrm{~h} \text { after surgery } \\
(\mathrm{mL})\end{array}$ & $\begin{array}{l}530.0(300.0 \\
\quad 900.0)\end{array}$ & $\begin{array}{l}500.0(300.0 \\
850.0)\end{array}$ & $\begin{array}{c}630.0(300.0 \\
1,100.0)\end{array}$ & 0.025 & $\begin{array}{l}500.0(330.0 \\
850.0)\end{array}$ & $\begin{array}{l}600.0(285.0 \\
985.0)\end{array}$ & 0.195 \\
\hline Ventilation time (hour) & $17.0(11.0,43.0)$ & $16.0(11.0,39.1)$ & $22.0(12.9,58.8)$ & 0.001 & $18.1(11.5,47.3)$ & $22.0(13.0,59.0)$ & 0.204 \\
\hline ICU Stay time (day) & $5.0(3.0,7.0)$ & $4.0(3.0,7.0)$ & $6.0(3.0,10.0)$ & $<0.001$ & $4.0(3.0,6.0)$ & $5.0(3.0,8.0)$ & 0.010 \\
\hline Hospital stay time (day) & $21.3 \pm 12.3$ & $21.1 \pm 11.5$ & $22.1 \pm 14.3$ & 0.302 & $22.0 \pm 12.2$ & $22.5 \pm 14.2$ & 0.646 \\
\hline 30-day mortality (\%) & $114(11.6)$ & $60(8.4)$ & $54(20.1)$ & $<0.001$ & $30(11.5)$ & $47(18.1)$ & 0.006 \\
\hline
\end{tabular}

Notes: Data presented as $\mathrm{n}(\%)$, median (IQR), or mean \pm standard deviation. $\mathrm{P}$ values indicate differences between non-HD patients and $H D$ patients. $P<0.05$ was considered statistically significant. PSM, propensity score matching; MELD, Model of End-Stage Liver Disease; CABG, coronary artery bypass graft; CPB, cardiopulmonary bypass; DHCA, deep hypothermic circulatory arrest; ICU, intensive care unit; $\mathrm{HD}$, hepatic dysfunction. 
Table 3 Multivariate analysis of risk factors for preoperative hepatic dysfunction

\begin{tabular}{lccc}
\hline Variables & OR & $95 \% \mathrm{Cl}$ & $\mathrm{P}$ value \\
\hline Age & 1.009 & $0.994-1.024$ & 0.267 \\
Male & 0.933 & $0.599-1.454$ & 0.759 \\
BMI & 0.968 & $0.932-1.006$ & 0.096 \\
DeBakey type I aortic & 1.022 & $0.635-1.645$ & 0.929 \\
dissection & & & \\
$\begin{array}{l}\text { Previous cardiovascular } \\
\text { disease }\end{array}$ & 1.218 & $0.418-3.554$ & 0.718 \\
Previous cardiac surgery & 1.129 & $0.462-2.760$ & 0.790 \\
TnT & 1.884 & $1.395-2.544$ & $<0.001$ \\
Pericardial tamponade & 2.000 & $1.299-3.018$ & 0.002 \\
sCr & 1.045 & $1.037-1.054$ & $<0.001$ \\
\hline
\end{tabular}

$\mathrm{P}<0.05$ was considered statistically significant. OR, odds ratio; $\mathrm{Cl}$, confidence interval; BMI, body mass index; $\mathrm{TnT}$, troponin $\mathrm{T}$; $\mathrm{SCr}$, serum creatinine.

Table 4 Multivariate analysis of risk factors for operative mortality

\begin{tabular}{lccc}
\hline Variables & OR & $95 \% \mathrm{Cl}$ & P value \\
\hline Age & 1.050 & $0.993-1.111$ & 0.085 \\
MELD score & 0.984 & $0.886-1.093$ & 0.765 \\
Limb ischemia & 4.590 & $1.003-21.008$ & 0.050 \\
Cerebral ischemia & 0.389 & $0.089-1.706$ & 0.211 \\
Coronary ischemia & 32.630 & $6.283-169.450$ & $<0.001$ \\
Mesenteric ischemia & 3.027 & $0.665-13.778$ & 0.152 \\
Pericardial tamponade & 3.467 & $0.819-14.687$ & 0.091 \\
CPB time & 0.999 & $0.992-1.006$ & 0.759 \\
\hline
\end{tabular}

$\mathrm{P}<0.05$ was considered statistically significant. OR, odds ratio; $\mathrm{Cl}$, confidence interval; MELD, Model of End-Stage Liver Disease; CPB, cardiopulmonary bypass.

The general health status of patients was evaluated annually by telephone consultation starting from 2014. By December 2020, 93.9\% (812 of 865) of all participating patients had been followed up for a median of 20 months. During the follow-up, 34 patients in the non-HD group and 17 in the HD group died. No significant differences were observed in the Kaplan-Meier survival curves after hospital deaths were excluded ( $\mathrm{P}=0.259$ by log-rank test; Figure 4$)$.
Table 5 Multivariate analysis of risk factors for postoperative 30-day mortality

\begin{tabular}{llll}
\hline Variables & OR & $95 \%$ Cl & P value \\
\hline Age & 2.586 & $1.006-6.650$ & 0.049 \\
Male & 1.032 & $0.996-1.070$ & 0.083 \\
BMI & 0.977 & $0.890-1.072$ & 0.622 \\
MELD score & 1.128 & $1.029-1.236$ & 0.010 \\
Pericardial tamponade & 1.632 & $0.342-7.785$ & 0.539 \\
Previous cardiovascular disease 3.630 & $0.491-26.834$ & 0.206 \\
Previous cardiac surgery & 2.239 & $0.200-25.112$ & 0.513 \\
Limb ischemia & 5.076 & $1.960-13.143$ & 0.001 \\
Cerebral ischemia & 2.212 & $0.196-24.958$ & 0.521 \\
Coronary ischemia & 12.888 & $4.581-36.260$ & $<0.001$ \\
Mesenteric ischemia & 7.028 & $2.894-17.067$ & $<0.001$ \\
WBC & 0.995 & $0.954-1.038$ & 0.819 \\
CRP & 0.997 & $0.989-1.005$ & 0.480 \\
D-dimer & 1.017 & $0.997-1.038$ & 0.089 \\
PLT & 1.003 & $0.996-1.010$ & 0.361 \\
CPB duration & 1.005 & $0.998-1.012$ & 0.183 \\
DHCA duration & 1.003 & $0.963-1.045$ & 0.877 \\
Postoperative AKI & 0.664 & $0.205-2.149$ & 0.494 \\
\hline P<0.05 was considered & & &
\end{tabular}

$\mathrm{P}<0.05$ was considered statistically significant. $\mathrm{OR}$, odds ratio; $\mathrm{Cl}$, confidence interval; BMI, body mass index; MELD, Model of End-Stage Liver Disease; WBC, white blood cell; PLT, platelet; CRP, c-reactive protein.

\section{Discussion}

This study suggested that preoperative HD, defined by the MELD score, is associated with increased in-hospital complications and mortality rates among patients who received surgical repair for ATAAD (after important confounding factors were adjusted for). Moreover, preoperative pericardial tamponade, elevated serum creatinine levels, and TnT levels upon admission were identified as independent predictors of preoperative HD. In addition, the preoperative MELD score was determined as a reliable predictive prognostic factor for 30-day morbidity after aortic operations.

Even though emergency surgery is an effective treatment for ATAAD, the mortality rate remains relatively high. 


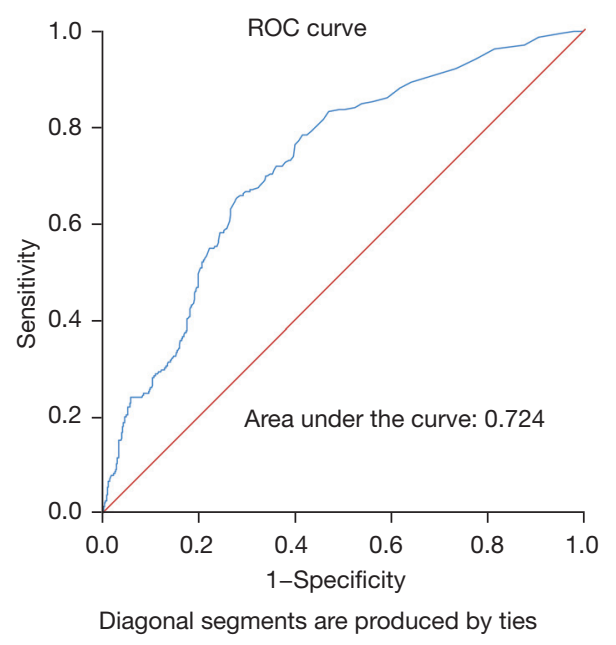

Figure 2 Receiver operating characteristics curve for determination of the cutoff value for prognostic serum troponin $T$ value upon admission in predicting preoperative hepatic dysfunction in acute type A aortic dissection.

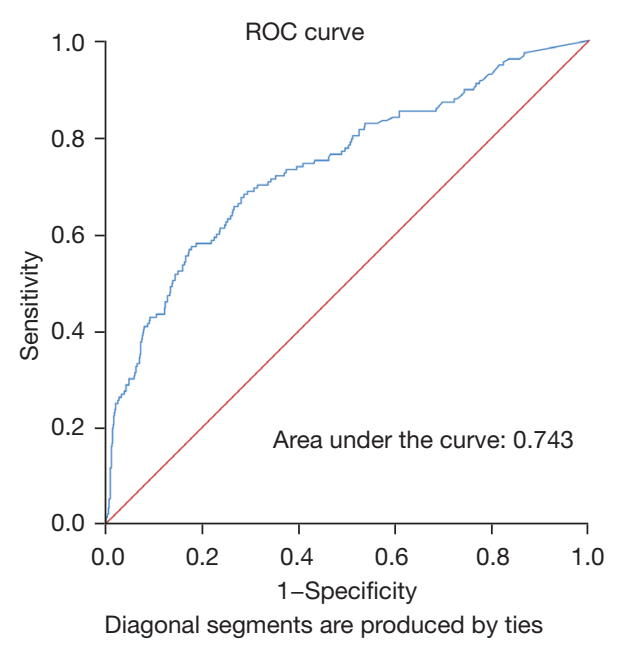

Figure 3 Receiver operating characteristics curve for determination of the cutoff value for MELD score upon admission in predicting postoperative dialysis in acute type A aortic dissection. MELD, Model of End-Stage Liver Disease.

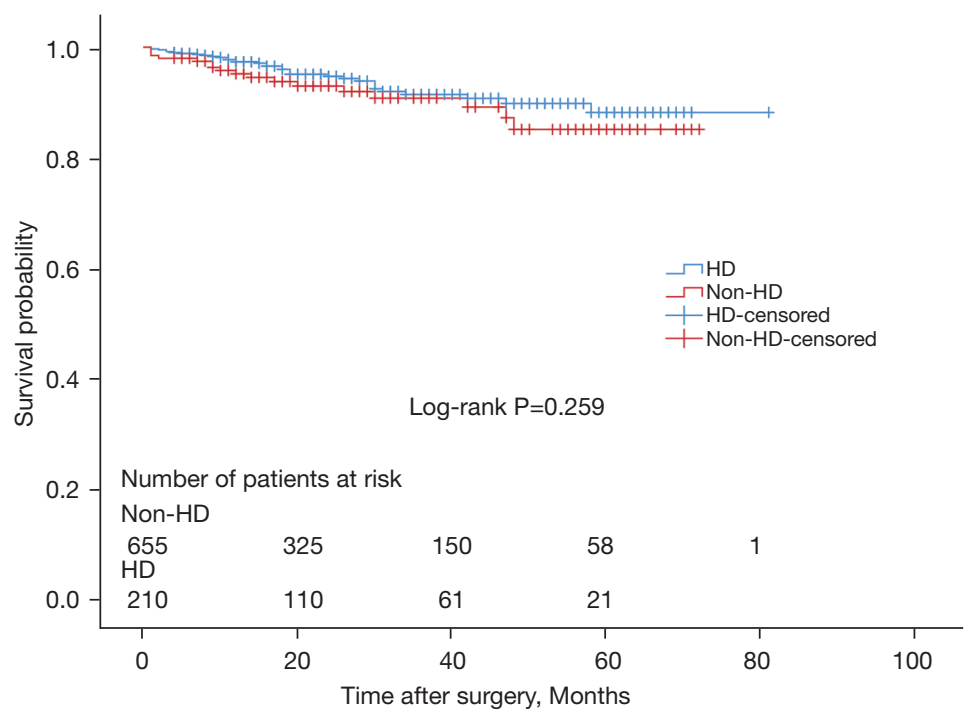

Figure 4 Kaplan-Meier survival curve of preoperative hepatic dysfunction in all patients with acute type A aortic dissection treated with surgical repair. HD, hepatic dysfunction.

Postoperative HD is a complication commonly observed after thoracic aortic surgery and is associated with increased in-hospital complications (9). However, there have been limited investigations regarding the incidence and prognostic value of preoperative $\mathrm{HD}$ in patients with ATAAD. In the present study, $27.2 \%$ of all enrolled patients were diagnosed with preoperative HD based on their MELD scores, and this was consistent with the
$22.4 \%$ incidence rate reported in a previous study involving patients who received coronary artery bypass grafting surgery or valvular surgery (10). However, the incidence was relatively low compared to the incidence of postoperative HD after aortic surgery (60.9\%) (9). Our data suggested that patients with preoperative HD were more likely to suffer from in-hospital complications compared to those without HD (69.8\% vs. $51.0 \%)$. Furthermore, contrary 
to previous studies (10), we demonstrated that patients with HD experienced more perioperative bleeding and prolonged respiratory support, both of which are indicators of poor outcomes. These results may partially explain why patients with preoperative HD had higher 30-day mortality rates compared to patients without preoperative HD.

Interestingly, our study showed that, although preoperative HD was associated with higher rates of 30-day mortality, it did not significantly affect long-term survival. This agrees with a previous study in which the survival and quality of life were not affected by HD in patients with infective endocarditis (15). However, little is known about the association between long-term survival and HD in patients with ATAAD. The MELD score measured in the study was not an indicator of baseline hepatic function, but rather a representation of the degree of circulatory compromise due to the aortic dissection. Once the aorta is repaired, circulatory blood flow is normalized, and the liver function (and consequently, the MELD score) should recover. This may explain why the long-term outcomes in survivors were not affected by HD as suggested in the study. Longer follow-up periods with hepatic function tests are necessary to verify this conclusion.

This study also aimed to determine whether the MELD score could be used to identify HD in ATAAD patients. The data suggested that elevated MELD scores were associated with poor postoperative outcomes. Furthermore, preoperative HD was accompanied by significantly higher drainage volume 24 hours after surgery and more frequent reoperation due to bleeding $(\mathrm{P}<0.001)$. The increased bleeding might be explained by a deficiency in coagulation factors in preoperative HD patients. Patients with high MELD scores due to high INR and liver dysfunction are more likely to develop perioperative coagulation abnormalities, and preoperative strategies such as infusion of plasma or clotting factors should thus be considered.

After baseline variables were adjusted for, multivariate analyses showed that cardiac tamponade upon admission was an independent predictor of preoperative HD. The incidence of cardiac tamponade was reported in $8-31 \%$ of patients with ATAAD (16-19). Cardiac tamponade is one of the most severe conditions in ATAAD, and right heart failure secondary to cardiac tamponade can result in acute liver dysfunction. In addition, reduction of hepatic blood flow due to decreased cardiac function can further contribute to the development of HD (20). Low blood pressure on admission was associated with reduced visceral perfusion, which causes generalized ischemia to the liver that may eventually lead to HD. Extension of the dissection may involve the visceral arteries and cause thrombosis or stenosis, which may directly decrease blood supply to the organs thereby resulting in acute injury. However, in our study, celiac axis or superior mesenteric artery involvement was not identified as a risk factor for preoperative HD. This could be explained by cardiac tamponade-induced venous hypertension and poor left ventricular filling, which leads to systemic arterial hypotension and an eventual decrease in the transhepatic pressure gradient, which in turn, results in a congested liver with inadequate oxygen delivery.

This study also identified elevated serum TnT as a risk factor for preoperative HD. TnT is a regulatory protein for myocardium contraction and a biochemical marker of myocardial injury and necrosis; it remains the preferred cardiac marker for the diagnosis of acute myocardial infarction (AMI) (21). Approximately 7\% of patients with aortic dissection have concomitant AMI (22). Acute cardiogenic HD is often associated with acute cardiocirculatory failure resulting from AMI and replacement of hepatocytes with red blood cells extravasating from the sinusoids and necrosis/apoptosis (23). Liver damage associated with impaired cardiac function has been reported as a complication of severe heart failure (24). Therefore, patients with elevated serum TnT levels upon admission due to AMI are more likely to develop preoperative HD due to acute cardiac insufficiency. Preoperative serum Cr level was also shown to be a risk factor for preoperative HD, suggesting that the presence of impaired renal function is an important determinant factor for developing deranged liver function tests.

The time-sensitive emergency treatment of aortic dissection means that strategies to improve hepatic perfusion (e.g., increasing the baseline blood pressure) are not feasible and may delay the surgery. In this case, prompt surgery is required to prevent the extension of the dissection and improve hepatic perfusion. Previous reports showed that a long cardiopulmonary bypass (CPB) duration $(>180$ minutes) is a strong risk factor for postoperative HD (25). Therefore, shorter CPB time would be desirable in preventing further liver impairment and improve in-hospital outcomes.

The present study demonstrated that the MELD score could predict the postoperative short-term outcomes. The greatest advantage of the MELD score is that it can assess HD easily and objectively. Wiesner et al. reported that the MELD score is an extremely powerful predictor of mortality in patients with chronic liver disease (26). 
Focusing on proper nutrition, inflammation, and HD to normalize MELD scores prior to surgery might be an effective way to improve postoperative clinical outcomes. In ATAAD patients, the MELD score might be a useful tool for assessing both the severity of the primary cause and the ischemia duration. This indicator may have good prognostic value in extreme situations such as cardiac tamponade or coronary malperfusion.

There were some limitations in this study. First, this was a single-center retrospective study, which might not be representative of the general population. Second, patients who died before admission were not included in the study, and some patients abandoned treatment and were prematurely discharged due to various reasons. Thus, inhospital mortality may be underestimated. Finally, due to the emergent nature of the disease, complete demographic data were not available for all patients.

\section{Conclusions}

In conclusion, preoperative HD was commonly observed among patients with ATAAD and was associated with increased in-hospital complications but did not affect long-term mortality. Furthermore, cardiac tamponade, elevated serum Cr levels and TnT levels were identified as independent predictors for preoperative HD.

\section{Acknowledgments}

Funding: None.

\section{Footnote}

Reporting Checklist: The authors have completed the STROBE reporting checklist. Available at https://dx.doi. org/10.21037/jtd-21-1051

Data Sharing Statement: Available at https://dx.doi. org/10.21037/jtd-21-1051

Conflicts of Interest: All authors have completed the ICMJE uniform disclosure form (available at https://dx.doi. org/10.21037/jtd-21-1051). The authors have no conflicts of interest to declare.

Ethical Statement: The authors are accountable for all aspects of the work in ensuring that questions related to the accuracy or integrity of any part of the work are appropriately investigated and resolved. The ethics committee of Nanjing Drum Tower Hospital approved this retrospective study (No. BL2014004) and waived the need for individual informed consent due to the nature of this study. The study was conducted in accordance with the Declaration of Helsinki (as revised in 2013).

Open Access Statement: This is an Open Access article distributed in accordance with the Creative Commons Attribution-NonCommercial-NoDerivs 4.0 International License (CC BY-NC-ND 4.0), which permits the noncommercial replication and distribution of the article with the strict proviso that no changes or edits are made and the original work is properly cited (including links to both the formal publication through the relevant DOI and the license). See: https://creativecommons.org/licenses/by-nc-nd/4.0/.

\section{References}

1. Howard DP, Banerjee A, Fairhead JF, et al. Populationbased study of incidence and outcome of acute aortic dissection and premorbid risk factor control: 10-year results from the Oxford Vascular Study. Circulation 2013;127:2031-7.

2. Beliaev AM, Bergin CJ. Impact of socioeconomic deprivation on incidence and outcomes of acute type A aortic dissection repair in New Zealand. J Card Surg 2021;36:2035-43.

3. Leontyev S, Tsagakis K, Pacini D, et al. Impact of clinical factors and surgical techniques on early outcome of patients treated with frozen elephant trunk technique by using EVITA open stent-graft: results of a multicentre study. Eur J Cardiothorac Surg 2016;49:660-6.

4. Inoue $\mathrm{Y}$, Minatoya K, Oda T, et al. Surgical outcomes for acute type A aortic dissection with aggressive primary entry resection. Eur J Cardiothorac Surg 2016;50:567-73.

5. Ziser A, Plevak DJ, Wiesner RH, et al. Morbidity and mortality in cirrhotic patients undergoing anesthesia and surgery. Anesthesiology 1999;90:42-53.

6. O'Leary JG, Friedman LS. Predicting surgical risk in patients with cirrhosis: from art to science. Gastroenterology 2007;132:1609-11.

7. Friedman LS. The risk of surgery in patients with liver disease. Hepatology 1999;29:1617-23.

8. O'Leary JG, Yachimski PS, Friedman LS. Surgery in the patient with liver disease. Clin Liver Dis 2009;13:211-31.

9. Zhou W, Wang G, Liu Y, et al. Outcomes and risk factors of postoperative hepatic dysfunction in patients 
undergoing acute type A aortic dissection surgery. J

Thorac Dis 2019;11:3225-33.

10. Murata M, Kato TS, Kuwaki K, et al. Preoperative hepatic dysfunction could predict postoperative mortality and morbidity in patients undergoing cardiac surgery: Utilization of the MELD scoring system. Int J Cardiol 2016;203:682-9.

11. Malinchoc M, Kamath PS, Gordon FD, et al. A model to predict poor survival in patients undergoing transjugular intrahepatic portosystemic shunts. Hepatology 2000;31:864-71.

12. Morisaki A, Hosono M, Sasaki Y, et al. Risk factor analysis in patients with liver cirrhosis undergoing cardiovascular operations. Ann Thorac Surg 2010;89:811-7.

13. Filsoufi F, Salzberg SP, Rahmanian PB, et al. Early and late outcome of cardiac surgery in patients with liver cirrhosis. Liver Transpl 2007;13:990-5.

14. Kim MS, Kato TS, Farr M, et al. Hepatic dysfunction in ambulatory patients with heart failure: application of the MELD scoring system for outcome prediction. J Am Coll Cardiol 2013;61:2253-61.

15. Diab M, Sponholz C, von Loeffelholz C, et al. Impact of perioperative liver dysfunction on in-hospital mortality and long-term survival in infective endocarditis patients. Infection 2017;45:857-66.

16. Rampoldi V, Trimarchi S, Eagle KA, et al. Simple risk models to predict surgical mortality in acute type A aortic dissection: the International Registry of Acute Aortic Dissection score. Ann Thorac Surg 2007;83:55-61.

17. Tsai TT, Evangelista A, Nienaber CA, et al. Longterm survival in patients presenting with type $\mathrm{A}$ acute aortic dissection: insights from the International Registry of Acute Aortic Dissection (IRAD). Circulation 2006;114:1350-6.

18. Hayashi T, Tsukube T, Yamashita T, et al. Impact of controlled pericardial drainage on critical cardiac tamponade with acute type A aortic dissection. Circulation 2012;126:S97-S101.

Cite this article as: Wang Z, Chen T, Ge M, Chen C, Lu L, Zhang L, Wang D. The risk factors and outcomes of preoperative hepatic dysfunction in patients who receive surgical repair for acute type A aortic dissection. J Thorac Dis 2021;13(10):5638-5648. doi: 10.21037/jtd-21-1051
19. Tan ME, Kelder JC, Morshuis WJ, et al. Risk stratification in acute type A dissection: proposition for a new scoring system. Ann Thorac Surg 2001;72:2065-9.

20. Kin Y, Nimura Y, Hayakawa N, et al. Doppler analysis of hepatic blood flow predicts liver dysfunction after major hepatectomy. World J Surg 1994;18:143-9.

21. Anderson JL, Adams CD, Antman EM, et al. 2012 ACCF/ AHA focused update incorporated into the ACCF/AHA 2007 guidelines for the management of patients with unstable angina/non-ST-elevation myocardial infarction: a report of the American College of Cardiology Foundation/ American Heart Association Task Force on Practice Guidelines. Circulation 2013;127:e663-828.

22. Hiratzka LF, Bakris GL, Beckman JA, et al. 2010 ACCF/AHA/AATS/ACR/ASA/SCA/SCAI/SIR/STS/ SVM guidelines for the diagnosis and management of patients with Thoracic Aortic Disease: a report of the American College of Cardiology Foundation/American Heart Association Task Force on Practice Guidelines, American Association for Thoracic Surgery, American College of Radiology, American Stroke Association, Society of Cardiovascular Anesthesiologists, Society for Cardiovascular Angiography and Interventions, Society of Interventional Radiology, Society of Thoracic Surgeons, and Society for Vascular Medicine. Circulation 2010;121:e266-369.

23. Çă̆lı K, Başar FN, Tok D, et al. How to interpret liver function tests in heart failure patients? Turk J Gastroenterol 2015;26:197-203.

24. Fouad YM, Yehia R. Hepato-cardiac disorders. World J Hepatol 2014;6:41-54.

25. Liu N, Sun LZ, Chang Q. The relative risk factors analysis of hepatic dysfunction following aortic dissection repair. Zhonghua Wai Ke Za Zhi 2010;48:1154-7.

26. Wiesner RH, McDiarmid SV, Kamath PS, et al. MELD and PELD: application of survival models to liver allocation. Liver Transpl 2001;7:567-80. 